\title{
Unswollen layer of cross-linked polyisoprene at the solid interface
}

\author{
Shinichiro Shimomura, ${ }^{a}$ Manabu Inutsuka, ${ }^{a} *$ Norifumi L. Yamada, ${ }^{b}$ and Keiji Tanaka
} $a, c, *$

a Department of Applied Chemistry, Kyushu University, Fukuoka 819-0395, Japan.

${ }^{\mathrm{b}}$ High Energy Accelerator Research Organization, Ibaraki 319-1106, Japan.

${ }^{\mathrm{C}}$ International Institute for Carbon-Neutral Energy Research (WPI-I2CNER), Kyushu University, Fukuoka 819-0395, Japan,

E-mail: m-inutsuka@cstf.kyushu-u.ac.jp (MI) and k-tanaka@cstf.kyushu-u.ac.jp (KT).

\begin{abstract}
The density profile in a thin cross-linked polyisoprene (PI) film spin-coated on a quartz substrate in $n$-hexane was examined by specular neutron reflectivity. We found two layers with different PI densities at the substrate interface. The characteristics of the layers are discussed in terms of bound rubber typically associated with the field of rubber materials and adsorbed layers typically associated with the field of glassy materials.
\end{abstract}

Keywords: Density profile, Adsorbed layer, Solid interface 


\section{Introduction}

Polymeric materials have often been used by mixing them with inorganic fillers to improve their physical performance including their mechanical properties and durability.[1-3] As an important example of these polymer composite materials, tires for motor vehicles have silica and/or carbon black nanoparticles dispersed in a cross-linked rubbery matrix made of natural rubber, polybutadiene (PB), butyl rubber and poly(styrene-co-butadiene) (SBR) with various stabilizers. Many studies have shown that the performance of tires is closely related to the interaction between the rubber matrix and the inorganic fillers.

In the field of rubber nanocomposites, it has been widely accepted for many years that rubber chains are tightly adsorbed on fillers and thus the chains have less mobility compared to when they are unbound as bulk chains. This "bound rubber" dissolves very little even in good solvents. In early 1970’s, pulse nuclear magnetic resonance (NMR) studies indicated that the molecular motion of chains is extremely suppressed in the bound rubber layer.[4-6] Other studies with NMR analysis, dielectric measurement or mechanical measurement proposed that there should exist two different kinds of chains in the interfacial bound rubber: "tightly adsorbed" and "loosely adsorbed" chains.[5,7-15] The former exhibits no clear glass-rubber transition, meaning that the chains are glassy even at room temperature. The latter shows moderate mobility, somewhere between that shown by the tightly adsorbed chains and that shown in bulk chains. A possible model to explain how rubber nanocomposites can be reinforced is that fillers with a bound rubber layer form a network structure in the matrix.[16-21] This notion is consistent with a very recent study by Mujtaba et al. dealing with the role of the bound rubber in the mechanical properties of rubber-silica nanocomposites.[22] 
Some research groups have conducted neutron scattering experiments on SBR-silica and PB-carbon black nanocomposites to explore the structure of the adsorbed interfacial layer on the fillers.[19,23]. However, more knowledge of the interfacial layer is required to better understand the bound rubber.

Over the last two decades, the segmental dynamics of polymer chains in a thin film supported on a solid substrate has been a hot topic in the field.[24-28] In the case of thin polystyrene (PS) films on silicon wafers with a native oxide layer, once the film becomes thinner than approximately $100 \mathrm{~nm}$, the glass transition temperature $\left(T_{\mathrm{g}}\right)$ starts to decrease with decreasing thickness.[24-28] This is mainly because the dynamics of the chains is faster in the surface region than in bulk.[29-35] At the same time, it is also accepted that chains form an adsorbed layer on the silicon surface[36-47] and exhibit slower dynamics.[48-50] These properties are similar to those of bound rubber, meaning that we should be able to explain how rubber and glassy polymers attach to solid surfaces in the same way.

The objective of this study is to discuss the aggregation states of cross-linked cis-1,4-polyisoprene, which is referred to as PI hereafter, at the interface with a quartz substrate on the basis of experimental techniques applied to thin polymer films. Specifically, the volume fraction of cross-linked PI in a thin spin-coated film swollen by a good solvent along the direction normal to the interface was examined by neutron reflectivity (NR), which is currently the most reliable method for investigating a thin layer with a resolution better than $1 \mathrm{~nm}$.[51,52] The information obtained will be useful to give a better understanding of the properties of bound rubber. 


\section{Experimental section}

\subsection{Materials}

PI was synthesized by living anionic polymerization using $n$-butyllithium as an initiator and methanol as a terminator. The number-average molecular weight $\left(M_{\mathrm{n}}\right)$ and molecular weight dispersity $\left(M_{\mathrm{w}} / M_{\mathrm{n}}\right)$, where $M_{\mathrm{w}}$ is the weight average molecular weight, of PI measured by gel permeation chromatography with polystyrene standards were $91 \mathrm{k}$ and 1.08 , respectively. The bulk $T_{\mathrm{g}}$ of PI by differential scanning calorimetry (DSC6220, Seiko Instrument, Inc.) was 211 K. Trimethylolpropane tris(3-mercaptopropionate) (TMMP) and phenylbis(2,4,6-trimethylbenzoyl)phosphine oxide (Irgacure 819) were used as a cross-linking agent and a photocleavable initiator, respectively. Solvents of hexane, $n$-hexane- $d_{14}$ and toluene were used without further purification. Chemicals, except for hexane (Kanto Chemical Co., Inc.) and toluene (Wako Pure Chemical Industries, Ltd.), were purchased from Sigma-Aldrich, Co. LCC. Fig. 1 shows chemical structures of PI, TMMP and Irgacure 819. They were all dissolved in toluene. The weight fractions of PI, TMMP and Irgacure 819 to toluene were $5,5 \times 10^{-2}$ and $2.5 \times 10^{-2}$ wt $\%$. Thin films of PI containing cross-linking agent and a photocleavable initiator were prepared by spin-coating from a solution onto quartz substrates or silicon wafers with a native oxide layer. Then, the films were irradiated with ultraviolet light with a wavelength of $365 \mathrm{~nm}$ and an intensity of about 100 $\mathrm{mW} \cdot \mathrm{cm}^{-2}$ for $15 \mathrm{~s}$ to promote the cross-linking reaction. The detailed reaction scheme is described elsewhere.[53] The films were eventually washed with toluene to eliminate unreacted chemicals. After drying, the film thickness was evaluated by ellipsometry (M150, JASCO Co.). 


\subsection{Measurements}

To examine the distribution of the cross-linking agents along the direction normal to the surface, dynamic secondary ion mass spectrometry (DSIMS) was performed using a SIMS 4000 (Aomika Analysetechnik GmbH). To attain a steady state sputtering for the sample, a sacrificed deuterated PS (dPS) film with a thickness of about $100 \mathrm{~nm}$ was deposited on the cross-linked PI film by a floating method. Then, a gold layer was deposited on the film to avoid charge-up during the measurements. The incident beam of oxygen ions with a voltage of $4.0 \mathrm{kV}$ and a current of $35 \mathrm{nA}$ was focused onto a 400 $\mu \mathrm{m} \times 400 \mu \mathrm{m}$ area of the sample surface. NR measurements were performed on the Soft Interface Analyzer (SOFIA at beam line No.16 in the Materials and Life Science Facility, Japan Proton Accelerator Research Complex, Tokai, Japan).[54,55] Neutron beams were introduced onto the film from the air side for measurements in air, and from the quartz side for measurements in $n$-hexane- $d_{14}$. NR data were then collected as a function of scattering vector $q$ given by $(4 \pi / \lambda) \cdot \sin \theta$, where $\lambda$ and $\theta$ are the wavelength and the incident angle of the neutrons, respectively. The analyses of NR data were carried out using Motofit software. [56] The scattering length density $(b / V)$ values for quartz, PI and $n$-hexane- $d_{14}$ were taken as $3.48 \times 10^{-4}, 0.27 \times 10^{-4}$ and $6.14 \times 10^{-4} \mathrm{~nm}^{-2}$, respectively. Reflectivity (XR) measurements using X-rays with a wavelength of $0.154 \mathrm{~nm}$ were also made for a non-cross-linked PI film on a silicon wafer, which was rinsed 3 times in toluene for 10 min each time, so that the thickness of the adsorbed PI layer on the substrate could be analyzed. The obtained XR data were analyzed with Parratt32 software based on Parratt's algorithm. Ellipsometry was also conducted 
using a Xe light source with a wavelength of $633 \mathrm{~nm}$. The surface morphology and thickness of the sample film in air and in hexane were examined by atomic force microscopy (AFM, E-sweep with an SPI3800 controller, SII Nano Technology Inc.) in dynamic force mode at room temperature. Cantilevers with a spring constant of 1.6 $\mathrm{N} \cdot \mathrm{m}^{-1}$ and a resonance frequency of $28 \mathrm{kHz}$ were used. The measurements were conducted in air, and in $n$-hexane after immersing the sample film in $n$-hexane for $12 \mathrm{~h}$.

\section{Results and Discussion}

The density profile of a thin cross-linked PI film spin-coated on a quartz substrate along the direction normal to the interface was examined by NR. Panel (a) in Fig. 2 shows NR curves for the PI film in air and in $n$-hexane, which is typically a good solvent of PI. To create a contrast at the PI/hexane interface, deuterated $n$-hexane was used in this test. The curve acquired in air exhibited clear Kiessig fringes resulting from constructive and destructive interference of neutron beams reflected from the air and quartz interfaces. On the other hand, the curve in $n$-hexane- $d_{14}$ showed a narrower fringe in a low- $q$ region. Once $q$ went beyond $0.4 \mathrm{~nm}^{-1}$, the fringes disappeared. An intuitive interpretation of these results is that the PI film was swollen, leading to a thickening of the film and a diffusing of the hexane interface.

To confirm this hypothesis, NR curves were fitted by a layer model. The best fit reflectivity curves were obtained using model depth profiles of $(b / V)$ shown in panel (b) of Fig. 2. For the curve fits of both air and $n$-hexane- $d_{14}$, the mass conservation of PI was considered. For the PI film in air, the thickness estimated was $44.1 \mathrm{~nm}$ using a simple 1-box model with interfacial roughness. This value was in good accordance with the results by XR and AFM. It is noteworthy that $(b / V)$ was constant through the 
PI film. The $(b / V)$ values of TMMP and Irgacure819 were $0.87 \times 10^{-4}$ and $1.58 \times 10^{-4}$ $\mathrm{nm}^{-2}$, respectively, and larger than the value of $0.27 \times 10^{-4} \mathrm{~nm}^{-2}$ for PI. Thus, it seems most likely that the cross-linking agents were homogeneously distributed along the normal direction in the film. This is discussed later based on DSIMS measurements.

When the curve for the cross-linked PI film in $n$-hexane- $d_{14}$ was fitted, a 3-layer model with respect to $(b / V)$ was adopted, as shown in panel (b) of Fig. 2. This was because the 1- and 2-layer(s) model did not fit the experimental data well. Fig. 3 shows the fitting results using 1- and 2-layer(s) models. The fitting parameters for the 3-layer model are shown in Table 1. The thickness of the film increased from 44.1 to $67.0 \mathrm{~nm}$. This change was also confirmed by AFM in $n$-hexane, as shown in Fig. S1, although the thickness measured was slightly lower $(\sim 64 \mathrm{~nm})$. This is probably because the swollen film was quite soft and was pushed down by the AFM tip. Alternatively, since the PI/hexane interface is quite ill-defined, there would be variability in the measurements. The $(b / V)$ value of the film also increased due to the penetration of $n$-hexane- $d_{14}$. As marked in Fig. 2(b), the film can be divided into the three layers: a ca. 4 nm-thick interfacial layer with quartz, a ca. 11 nm-thick intermediate layer, and a bulk layer.

The $(b / V)$ value of the interfacial layer was almost the same as that of the pure PI, meaning that chains near the substrate did not swell much, even when a good solvent was used. We believed that the interfacial layer was associated with "tightly adsorbed" bound rubber, or a "dead layer”, as suggested in many previous reports. If that was the case, the thermal mobility of chains in the interfacial layer would be suppressed by the presence of the substrate. For example, using pulsed NMR, Nishi found that the spin-spin relaxation time in the bound rubber layer for a system of natural rubber and 
carbon black remained unchanged even in $\mathrm{CCl}_{4}$.[4] This finding tells us that rubbery chains in the bound rubber layer do not swell much, even in a good solvent. Similar results were also reported by many other groups.[4,8,9,11,14,57,58] Why chains at the solid interface do not swell much, even in a good solvent, can be explained in terms of thermodynamics; the number of contacts between chain segments and the substrate surface does not decrease.[46]

There existed an intermediate layer, which was less swollen than the bulk region, between the interfacial layer and bulk region. Assuming a simple additivity rule without allowing for density change, the volume fraction $(\phi)$ of PI is given by:

$$
(b / V)=(b / V)_{\mathrm{PI}} \cdot \phi+(b / V)_{\mathrm{dhex}} \cdot(1-\phi)
$$

where the subscripts PI and dhex are PI and $n$-hexane- $d_{14}$, respectively. The $\phi$ in the intermediate layer ranged from 0.54 to 0.84 , which is significantly higher than that in bulk. Takenaka et al. studied the same issue for SBR with silica particles in hexane by contrast variation small angle neutron scattering. They found an interfacial dense layer with a polymer volume fraction of 0.61 in hexane.[19] Our result is in good accordance with their results. Hence, the presence of the intermediate layer is not unique in our current system. Considering the density profile of this intermediate layer that is between the interfacial "tightly adsorbed" layer and the bulk layer, it seems most likely that the intermediate layer is composed of "loosely adsorbed" chains, which can be swollen to some degree, as mentioned in previous reports.[59]

PI swelled more in the bulk layer than in the interfacial adsorbed layers, in which chains are loosely adsorbed onto the quartz substrate. In this case, the existence probability of PI segments should decrease with a distance from the quartz interface. 
On the other hand, this was not the case in the bulk layer. The network in the bulk homogeneously swells. The $\phi$ in this layer was calculated to be about 0.53 . Postulating a 1-dimensional swelling model for the thin film, where there is no swelling along the direction parallel to the interface, a modified Flory-Rehner equation was used to determine the cross-linking density in the layer.[60-62]

$$
v=\left\{\ln (1-\phi)+\phi+\chi \phi^{2}\right\} /\left\{\frac{1}{2} V_{\text {dhex }}(\phi-2)\right\}
$$

where $v$ is the cross-linking density, $V_{\text {dhex }}$ is the molar volume of $n$-hexane- $d_{14}$, and $\chi$ is the Flory-Huggins interaction parameter for PI with hexane. $V_{\text {hex }}$ and $\chi$ values of $1.32 \times 10^{-4} \mathrm{~m}^{3} \cdot \mathrm{mol}^{-1}$ and 0.47 were used, respectively,[63] to calculate the $v$ value of $9.3 \times 10^{4} \mathrm{~mol} \cdot \mathrm{m}^{-3}$. From this number, the average molecular weight of PI between cross-linking points is estimated to be about $1.0 \times 10^{3}$. That is, there exist 22 isoprene monomers between the cross-linking points. This value is similar to the expected value of 36 monomers based on the stoichiometric ratio between PI and the cross-linking agent.

The distribution of cross-linking agents along the direction normal to the interface through the film should be confirmed. Panel (a) of Fig. 4 shows the SIMS depth profiles of $\mathrm{H}^{-}, \mathrm{D}^{-}, \mathrm{C}^{-}, \mathrm{Si}^{-}, \mathrm{P}^{-}$and $\mathrm{S}^{-}$in a $\mathrm{PI}$ film with cross-linking agents. $\mathrm{D}^{-}$from the sacrificed layer of dPS and $\mathrm{Si}^{-}$are markers that define the surface and substrate interface, respectively. Panel (b) of Fig. 4 shows concentration profiles of TMMP and Irgacure819, which were calculated on the basis of the etching time dependence of $\mathrm{S}^{-}$ and $\mathrm{P}^{-}$, respectively.[64] At a glance, the concentrations of TMMP and Irgacure819 are almost constant through the film. It is noteworthy that their values correspond well with the initial amounts fed. However, careful reading of Fig. 4(b) reveals that the 
concentrations of TMMP are only slightly lower at the surface and slightly higher at the substrate interface than in the internal region of the film. That is, TMMP with a surface free energy higher than PI is depleted and segregated at the surface and substrate interface. However, the extents of the surface segregation and interfacial depletion of TMMP were less than 0.5 vol\%. Hence, the cross-linking density may show little or no change at the surface and substrate interface.

The relation between bound rubber and tightly adsorbed chains at the substrate interface is discussed further. Here, a PI film without cross-linking was similarly spin-coated on a quartz substrate and then thoroughly washed. The thickness of the residual adsorbed PI layer was examined. Fig. 5 shows XR curves for the reference substrate and PI adsorbed layer. Fitting with a simple layer model revealed that the thickness of the layer of adsorbed PI chains after sufficient washing was $1.1 \mathrm{~nm}$. This result was well supported by independent AFM and ellipsometry measurements. A point, which should be emphasized, is that the thickness of the interfacial bound rubber layer seen in Fig. 2 is larger than that of the tightly adsorbed layer from the film without cross-linking in Fig. 5. The difference in the two films shown in Figs. 2 and 5 is due to the presence of cross-linking. Since the molecular weight of the PI chains effectively becomes larger after the cross-linking reaction, the adsorbed layer should be commensurately thicker.[37]

In conclusion, we applied NR measurements to a cross-linked PI film on a quartz substrate in a good solvent and found that it heterogeneously swelled along the direction normal to the interface. There exists an interfacial layer with little swelling and an intermediate layer with some swelling at the substrate interface. These layers are related to descriptions of bound rubber mentioned in previous reports. We also 
characterized a non-cross-linked PI film on the substrate after washing it with a good solvent using XR. The residual adsorbed layer was thinner than the layer showing little swelling. This implies that the aggregation states and thus the mechanical properties of bound rubber should be controllable by cross-linking the rubber chains.

\section{ASSOCIATED CONTENT}

Supporting Information. Supplementary data related to this article can be found at http://...

\section{Acknowledgements}

This work was partly supported by Young Scientists (B) (No. 15K17871) and Scientific Research (A) (no. 15H02183) from the Ministry of Education, Culture, Sports, Science and Technology, Japan. We are also grateful for support from the Japan Science and Technology Agency (JST) SENTANKEISOKU (13A0004) and Impulsing Paradigm Change through Disruptive Technologies Program (ImPACT). NR measurements were performed on BL-16 at the Materials and Life Science Facility, J-PARC, Japan, under Program No. 2009S08, 2014 S08 and 2016A0217. 


\section{References}

[1] V. Ganesan, A. Jayaraman, Soft Matter 10 (2014) 13-38.

[2] N. S. Enikolopyan, M. L. Fridman, I. O. Stalnova, V. L. Popov, Adv. Polym. Sci. 96 (1990) 1-67.

[3] B. Alcock, T. Peijs, Adv. Polym. Sci. 251 (2013) 1-76.

[4] T. Nishi, J. Polym. Sci.: Polym. Phys. Ed. 12 (1974) 685-693.

[5] J. O'Brien, E. Cashell, G. E. Wardell, V. J. McBrierty, Macromolecules 9 (1976) 653-660.

[6] J. Van Alsten, Macromolecules 24 (1991) 5320-5323.

[7] S. Kaufman, W. P. Slichter, D. D. Davis, J. Polym. Sci. Part A-2: Polym. Phys. 9 (1971) 829-839.

[8] M. Ito, T. Nakamura, K. Tanaka, J. Appl. Polym. Sci. 30 (1985) 3493-3504.

[9] A. P. Legrand, N. Lecomte, A. Vidal, B. Haidar, E. Papirer, J. Appl. Polym. Sci. 46 (1992) 2223-2232.

[10] K. U. Kirst, F. Kremer, V. M. Litvinov, Macromolecules 26 (1993) 975-980.

[11] J. W. ten Brinke, V. M. Litvinov, J. E. G. J. Wijnhoven, J. W. M. Noordermeer, Macromolecules 35 (2002) 10026-10037.

[12] A. Papon, K. Saalwächter, K. Schäler, L. Guy, F. Lequeux, H. Montes, Macromolecules 44 (2011) 913-922.

[13] A. Papon, H. Montes, M. Hanafi, F. Lequeux, L. Guy, K. Saalwächter, Phys. Rev. Lett. 108 (2012) 065702.

[14] Z. Jun-Xue, W. He, S. Xin-Yan, Z. Shu-Gao, J. Macromol. Sci., Part B 51 (2012) 496-509.

[15] S.-S. Choi, E. Ko, Polym. Test. 40 (2014) 170-177.

[16] Y. Fukahori, J. Appl. Polym. Sci. 95 (2005) 60-67.

[17] T. Koga, M. Takenaka, K. Aizawa, M. Nakamura, T. Hashimoto, Langmuir 21 (2005) 11409-11413.

[18] A. A. Gusev, Macromolecules 39 (2006) 5960-5962.

[19] M. Takenaka, S. Nishitsuji, N. Amino, Y. Ishikawa, D. Yamaguchi, S. Koizumi, Macromolecules 42 (2009) 308-311.

[20] H. Montes, T. Chaussée, A. Papon, F. Lequeux, L. Guy, Eur. Phys. J. E 31 (2010) 263-268.

[21] I. Morozov, B. Lauke, G. Heinrich, Comp. Mater. Sci. 47 (2010) 817-825.

[22] A. Mujtaba, M. Keller, S. Ilisch, H. J. Radusch, M. Beiner, T. Thurn-Albrecht, K. Saalwächter, ACS Macro Lett. 3 (2014) 481-485. 
[23] N. Jiang, M. K. Endoh, T. Koga, T. Masui, H. Kishimoto, M. Nagao, S. K. Satija, T. Taniguchi, ACS Macro Lett. 4 (2015) 838-842.

[24] R. A. L. Jones, Curr. Opin. Colloid Int. Sci. 4 (1999) 153-158.

[25] R. Inoue, K. Kawashima, K. Matsui, T. Kanaya, K. Nishida, G. Matsuba, M. Hino, Phys. Rev. E 83 (2011) 021801.

[26] C. Zhang, Y. Fujii, K. Tanaka, ACS Macro Lett. 1 (2012) 1317-1320.

[27] Y. Fujii, H. Morita, A. Takahara, K. Tanaka. Adv. Polym. Sci. 252 (2013) $1-27$.

[28] R. Inoue, T. Kanaya, Adv. Polym. Sci. 252 (2013) 107-140.

[29] J. L. Keddie, R. A. L. Jones, R. A. Cory, Faraday Discuss. 98 (1994) 219-230.

[30] J. A. Forrest, K. Dalnoki-Veress, J. R. Dutcher, Phys. Rev. E 56 (1997) 5705-5716.

[31] T. Kajiyama, K. Tanaka, A. Takahara, Macromolecules 30 (1997) 280-285.

[32] K. Tanaka, A. Takahara, T. Kajiyama, Macromolecules 33 (20) (2000) 7588-7593.

[33] K. Tanaka, K. Hashimoto, T. Kajiyama, A. Takahara, Langmuir 19 (2003) 6573-6575.

[34] T. Miyazaki, K. Nishida, T. Kanaya, Phys. Rev. E 69 (2004) 061803.

[35] P. A. O'Connell, G. B. McKenna, Science 307 (2005) 1760-1763.

[36] R. Inoue, T. Kanaya, K. Nishida, I. Tsukushi, M. T. F. Telling, B. J. Gabrys, M. Tyagi, C. Soles, W. l. Wu, Phys. Rev. E 80 (2009) 031802.

[37] Y. Fujii, Z. H. Yang, J. Leach, H. Atarashi, K. Tanaka, O. K. C. Tsui, Macromolecules 42 (2009) 7418-7422.

[38] Z. H. Yang, Y. Fujii, F. K. Lee, C. H. Lam, O. K. C. Tsui, Science 328 (2010) 1676-1679.

[39] S. Napolitano, M. Wübbenhorst, Nat. Commun. 2 (2011) 260.

[40] H. Tsuruta, Y. Fujii, N. Kai, H. Kataoka, T. Ishizone, M. Doi, H. Morita, K. Tanaka, Macromolecules 45 (2012) 4643-4649.

[41] P. Gin, N. Jiang, C. Liang, T. Taniguchi, B. Akgun, S. K. Satija, M. K. Endoh, T. Koga, Physical Review Letters 109 (26) (2012) 265501.

[42] R. Inoue, M. Nakamura, K. Matsui, T. Kanaya, K. Nishida, M. Hino, Phys. Rev. E 88 (2013) 032601.

[43] N. Jiang, J. Shang, X. Di, M. K. Endoh, T. Koga, Macromolecules 47 (2014) 2682-2689. 
[44] N. Jiang, M. K. Endoh, T. Koga, T. Masui, H. Kishimoto, M. Nagao, S. K. Satija, T. Taniguchi, ACS Macro Lett. 4 (2015) 838-842.

[45] M. Inutsuka, A. Horinouchi, K. Tanaka, ACS Macro Lett. 4 (2015) 1174-1178.

[46] M. Sen, N. Jiang, J. Cheung, M. K. Endoh, T. Koga, D. Kawaguchi, K. Tanaka, ACS Macro Lett. (2016) 504-508.

[47] S. Shimomura, M. Inutsuka, K. Tajima, M. Nabika, S. Moritomi, H. Matsuno, K. Tanaka, Polym. J. DOI:10.1038/pj.2016.58.

[48] K. Tanaka, Y. Tsuchimura, K. Akabori, F. Ito, T. Nagamura, Appl. Phys. Lett. 89 (2006) 061916.

[49] K. Tanaka, Y. Tateishi, Y. Okada, T. Nagamura, M. Doi, H. Morita, J. Phys. Chem. B 113 (2009) 4571-4577.

[50] D. Kawaguchi, Y. Tateishi, K. Tanaka, J. Non-Cryst. Solid. 407 (2015) 284-287.

[51] T. P. Russell, Mater. Sci. Rep. 5 (1990) 171-271.

[52] T. Koga, Y. Ji, Y. S. Seo, C. Gordon, F. Qu, M. H. Rafailovich, J. C. Sokolov, S. K. Satija, J. Polym. Sci. Part B: Polym Phys. 42 (2004) 3282-3289.

[53] C. Decker, T. Nguyen Thi Viet, J. Appl. Polym. Sci. 82 (2001) 2204-2216.

[54] N. L. Yamada, N. Torikai, K. Mitamura, H. Sagehashi, S. Sato, H. Seto, T. Sugita, S. Goko, M. Furusaka, T. Oda, M. Hino, T. Fujiwara, H. Takahashi, A. Takahara, Eur. Phys. J. Plus 126 (2011) 1-13.

[55] K. Mitamura, N. L. Yamada, H. Sagehashi, N. Torikai, H. Arita, M. Terada, M. Kobayashi, S. Sato, H. Seto, S. Goko, M. Furusaka, T. Oda, M. Hino, H. Jinnai, A. Takahara, Polym. J. 45 (2013) 100-108.

[56] A. Nelson, J. Appl. Cryst. 39 (2006) 273-276.

[57] Y. Zhang, S. Ge, B. Tang, T. Koga, M. H. Rafailovich, J. C. Sokolov, D. G. Peiffer, Z. Li, A. J. Dias, K. O. McElrath, M. Y. Lin, S. K. Satija, S. G. Urquhart, H. Ade, D. Nguyen, Macromolecules 34 (2001) 7056-7065.

[58] D. Fragiadakis, L. Bokobza, P. Pissis, Polymer 52 (2011) 3175-3182.

[59] P. G. de Gennes, Scaling Concepts in Polymer Physics, Cornell Univ. Press, 1979.

[60] K. Dušek, A. Choukourov, M. Dušková-Smrčková, H. Biederman, Macromolecules 47 (2014) 4417-4427.

[61] P. J. Flory, J. Rehner, J. Chem. Phys. 11 (1943) 521-526.

[62] R. Toomey, D. Freidank, J. Rühe, Macromolecules 37 (2004) 882-887. 
[63] J. Brandrup, E. H. Immergut, E. A. Grulke, Polymer Handbook, Wiley Interscience, 1999.

[64] D. Kawaguchi, K. Tanaka, N. Torikai, A. Takahara, T. Kajiyama, Langmuir 23 (2007) 7269-7275. 
Table 1. Parameters obtained from the fitting results shown in Fig. 2(b).

\begin{tabular}{|c|c|c|c|c|c|c|c|c|c|c|c|}
\hline \multirow{2}{*}{ Model } & \multicolumn{3}{|c|}{$h_{\mathrm{i}} / \mathrm{nm}$} & \multicolumn{3}{|c|}{$(b / V)_{\mathrm{i}} \cdot 10^{4} / \mathrm{nm}^{-2}$} & \multicolumn{4}{|c|}{$\sigma_{\mathrm{j}} / \mathrm{nm}$} & \multirow{2}{*}{$\chi^{2} \cdot 10^{3}$} \\
\hline & $h_{1}$ & $h_{2}$ & $h_{3}$ & $(b / V)_{1}$ & $(b / V)_{2}$ & $(b / V)_{3}$ & $\sigma_{1}$ & $\sigma_{2}$ & $\sigma_{3}$ & $\sigma_{4}$ & \\
\hline air & $44.1 \pm 0.2$ & - & - & $0.36 \pm 0.02$ & & - & $1.4 \pm 0.2$ & $1.3 \pm 0.1$ & - & - & 1.7 \\
\hline$n$-hexane- $d_{14}$ & $52.0 \pm 0.8$ & $11.2 \pm 0.7$ & $3.8 \pm 0.9$ & $3.10 \pm 0.09$ & $1.30 \pm 0.05$ & $0.36 \pm 0.03$ & $9.0 \pm 0.2$ & $7.2 \pm 0.4$ & $0.2 \pm 0.1$ & $1.3 \pm 0.1$ & 7.1 \\
\hline
\end{tabular}




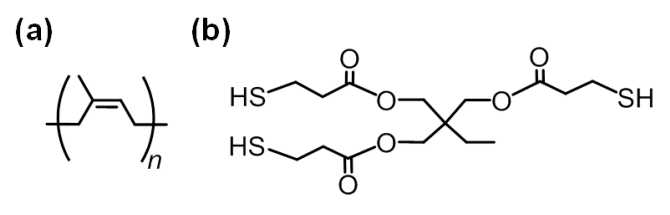

(c)

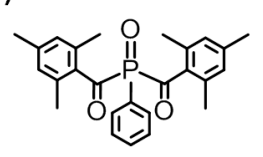

Fig. 1. Chemical structures of (a) PI, (b) TMMP and (c) Irgacure819. 

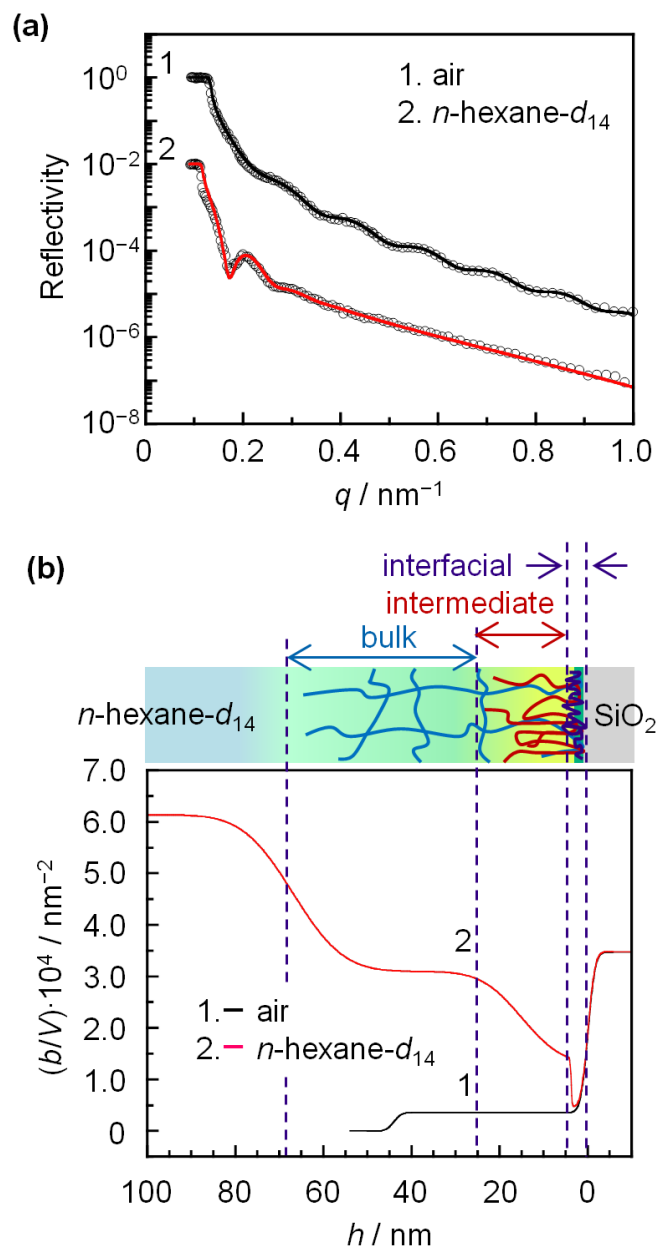

Fig. 2. (a) Neutron reflectivity curves for a cross-linked PI film in air and $n$-hexane- $d_{14}$. Open symbols depict experimental data and solid and dotted lines represent the reflectivity calculated on the basis of the scattering length density $(b / V)$ profiles shown in (b). 

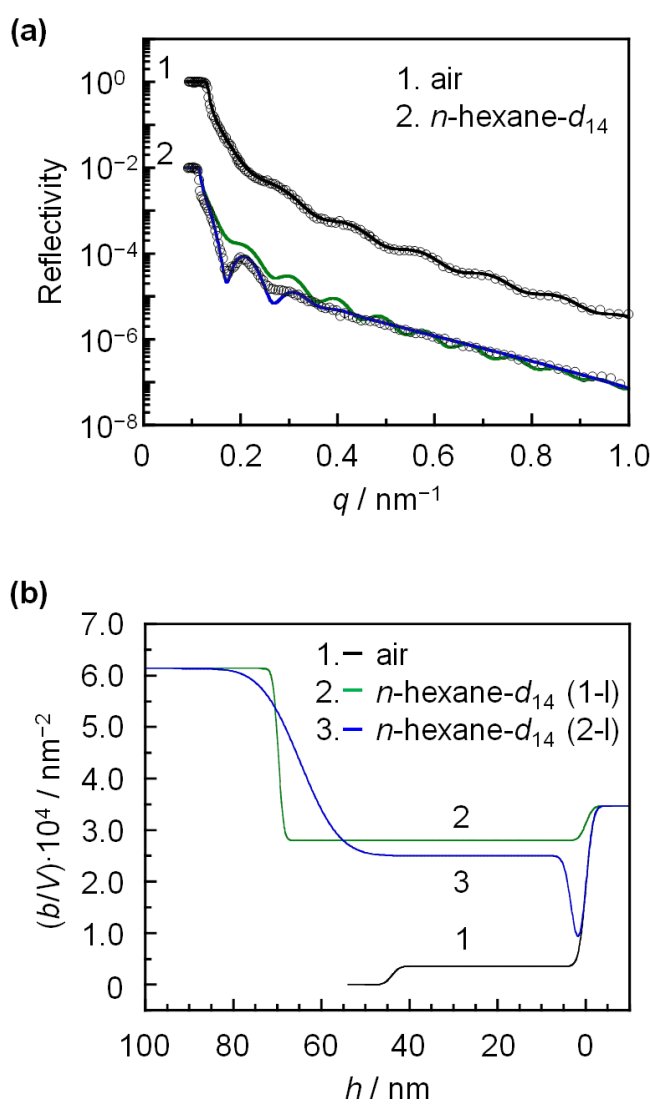

Fig. 3. (a) NR curves for a cross-linked PI film in air and $n$-hexane- $d_{14}$. Open symbols depict experimental data and solid lines represent the best-fit reflectivity calculated on the basis of the model $(b / V)$ profiles shown in (b). Experimental data are the same as those in Fig. 2. (a). As a fitting model in $n$-hexane, a single layer (1-l) and a double layer composed of interfacial and bulk layers (2-l) were used. 
(a)

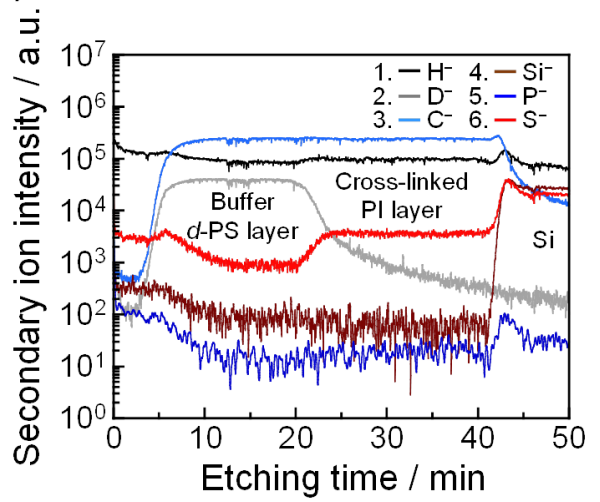

(b)

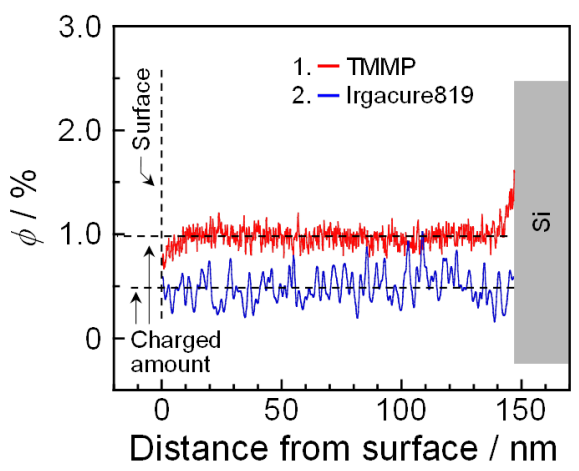

Fig. 4. (a) DSIMS profiles and (b) calculated depth profiles of volume fractions of cross-linking agents in a cross-linked PI film. 


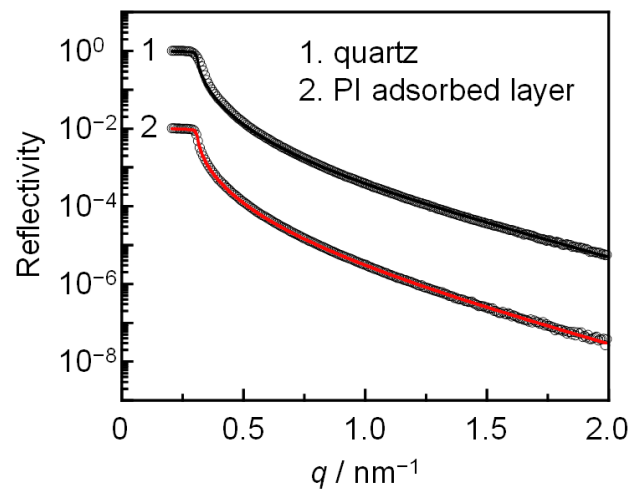

Fig. 5. XR curves for the quartz substrate and PI adsorbed layer. Symbols and solid lines denote experimental and calculated reflectivity, respectively. 
